\title{
ANALYTIC STRUCTURE IN SOME ANALYTIC FUNCTION ALGEBRAS
}

\author{
BY \\ WILLIAM R. ZAME $\left(^{1}\right)$
}

\begin{abstract}
A complete description is given of the analytic structure of maximal dimension in the spectra of a wide class of concrete function algebras generated by analytic functions. A connection is also given with point derivations on such algebras.
\end{abstract}

I. Introduction. Let $B$ be a function algebra acting on its spectrum (maximal ideal space) $\mathrm{Sp} B$, with Shilov boundary $\Gamma_{B}$. According to a wellknown result of Rossi [15], the functions in $B$ obey a weak maximum modulus principle on $\mathrm{Sp} B-\Gamma_{B}$. One of the principal themes in the theory of function algebras is the "explanation" of this analytic-type behavior by the existence of analytic structure in $\operatorname{Sp} B$. More precisely, let us say that an analytic variety (of dimension $n$ ) in $\operatorname{Sp} B$ is a pair $(X, \tau)$ where $X$ is an analytic variety (of pure dimension $n$ ), $\tau: X \rightarrow \operatorname{Sp} B$ is a continuous map, and $b \circ \tau$ is analytic for each $b \in B$. Then the question is: what are the nontrivial analytic varieties in $\operatorname{Sp} B$ ?

Although Stolzenberg's example [16] shows that one does not have the existence of nontrivial analytic structure in all situations, a number of global and local conditions which imply the existence of analytic structure have been found by Gleason [11], Browder [8], Clayton [9] and others. A different approach, due to Bishop [3], which depends upon the existence of functions in $B$ with finite fibers, leads to the construction of 1-dimensional analytic structure in $\operatorname{Sp} B$. Bishop's ideas have been expanded and exploited by Stolzenberg [17], Björk [5], [6], Alexander [1], [2], the author [20], and others, and have proven very useful in dealing with questions of approximation.

In this paper, we shall be concerned with the classification of high-dimensional analytic structure. For a fairly wide class of concrete function algebras, those which contain a sufficiently rich collection of analytic functions, we are able to completely describe the analytic structure of maximal dimension which

Received by the editors August 27, 1973 and, in revised form, February 6, 1974. AMS (MOS) subject classifications (1970). Primary 46J10; Secondary 32E25, 32E30.

Key words and phrases. Analytic function algebras, analytic structure, differentiably stable algebras, point derivations.

(1) Supported in part by NSF Grant PO37961 and by a grant from the Research Foundation, State University of New York. 
is present in the spectrum. In fact, the set of points in the spectrum through which an analytic variety of maximal dimension passes admits the structure of a complex analytic manifold, and the functions in the algebra are analytic on this manifold.

In §II, we collect some definitions and preliminary results; §III is devoted to the classification of maximal dimensional analytic structure, and §IV discusses some connections with point derivations. We make use of some of the ideas of Bishop [4] and a previous paper of the author [21] (which also contains a summary of the needed results from Bishop's paper); some knowledge of these papers will be helpful, but not really necessary. For general information about function algebras we refer to Gamelin [10] and Stout [18]; we use Gunning and Rossi [12] as a reference for the theory of several complex variables.

II. Preliminaries. Throughout, we let $S$ be a Stein manifold of dimension $n$ with global local coordinate $\sigma=\left(\sigma_{1}, \sigma_{2}, \cdots, \sigma_{n}\right)$; i.e., $(S, \sigma)$ is a Stein-Riemann domain. If $U$ is an open subset of $S$, then $O(U)$ denotes the algebra of functions analytic on $U$, endowed with the topology of uniform convergence on compact sets. For $V \subset U$, we let $r_{U V}: O(U) \rightarrow O(V)$ denote the restriction. If $K$ is a compact set, then $\left\{O(U), r_{U V}: K \subset V \subset U\right\}$ is an inductive system of topological algebras; we denote the inductive limit by $O(K)$. We may identify $O(K)$ as the algebra of germs on $K$ of functionals analytic near $K$; the topology is the finest which renders the natural maps $r_{U} ; O(U) \rightarrow$ $O(K)$ continuous. If $f$ is analytic near $K$, we denote its germ on $K$ by $f$; $f$ is a representative of $\mathbf{f}$. We sometimes abuse notation to consider an element of $O(K)$ as a complex-valued function on $K$; this should cause no confusion.

We denote differentiation with respect to the coordinate $\sigma$ by $D_{\sigma}$; thus, if $\alpha=\left(\alpha_{1}, \alpha_{2}, \cdots, \alpha_{n}\right)$ is a multi-index,

$$
D_{\sigma}^{\alpha}=\frac{\partial^{\alpha_{1}+\alpha_{2}+\cdots+\alpha_{n}}}{\partial \sigma^{\alpha_{1}} \cdots \partial \sigma_{n}^{\alpha_{n}}} .
$$

Observe that the operators $D_{\sigma}^{\alpha}: O(U) \rightarrow O(U)$ commute with the restrictions $r_{U V}$; thus they induce continuous operators on $O(K)$. We will say that a subalgebra $A$ of $O(K)$ is stable if

(i) f $\in A$ for each $f \in O(S)$, and

(ii) $D_{\sigma}^{\alpha} f \in A$ for each $f \in A$ and each multi-index $\alpha$.

If $B$ is a topological algebra, then $\operatorname{Sp} B$ denotes the spectrum (maximal ideal space) of $B$; i.e., the space of nonzero continuous, complex-valued homomorphisms of $B$, equipped with the weak-* topology. If $b \in B$, then $\hat{b}$, the Gelfand transform of $b$, is defined by $\hat{b}(\varphi)=\varphi(b)$ for each $\varphi \in \operatorname{Sp} B$. The map $b \mapsto \hat{b}$ represents $B$ as an algebra of continuous functions on $\operatorname{Sp} B$.

With each stable subalgebra $A$ of $O(K)$ we associate a function algebra 
$\tilde{A}$, which is the uniform closure in $C(K)$ of the image of $A$ under the natural map $O(K) \rightarrow C(K)$. Such algebras might be called stable function algebras. If $K$ is a compact subset of $\mathbf{C}^{n}$, then $P(K)$ (the closure in $C(K)$ of the polynomials), $R(K)$ (the closure in $C(K)$ of the rational functions with poles off $K$ ), and $H(K)$ (the closure in $C(K)$ of $O(K)$ ) are stable function algebras; $H(K)$ provides a particularly good model for what follows. The following proposition was proved in [21].

Proposition 1. If $A$ is a stable subalgebra of $O(K)$, then $\operatorname{Sp} A=$ Sp $\widetilde{A}$; in particular $\operatorname{Sp} A$ is compact.

In view of the above, the algebras $A$ and $\tilde{A}$ are essentially interchangeable for our purposes, since we are primarily concerned with the spectrum; it will usually be more convenient to work with $A$ rather than $\widetilde{A}$. Observe that the natural map $K \rightarrow \operatorname{Sp} A$ is an injection; we suppress the map and simply regard $K$ as a subset of $\operatorname{Sp} A$. Note that $\widetilde{A}^{\wedge}=\{\hat{g}: g \in \widetilde{A}\}$ is the uniform completion of $\hat{A}=\{\hat{\mathrm{f}}: \mathrm{f} \in A\}$. Thus the analytic structures in $\operatorname{Sp} A$ and $\operatorname{Sp} \tilde{A}$ are also identical.

We let $\hat{\sigma}=\left(\hat{\sigma}_{1}, \cdots, \hat{\sigma}_{n}\right): \operatorname{Sp} A \rightarrow \mathbf{C}^{n}$; this map plays a special role in what follows. In particular, we have the following result from [21].

Proposition 2. If $A$ is a stable subalgebra of $O(K)$ and $\zeta \in \mathbf{C}^{n}$, then $\hat{\sigma}^{-1}(\zeta)$ is totally disconnected, and hence the topological dimension of Sp $A$ does not exceed $2 n$.

In view of the above, there is certainly no analytic structure of (complex) dimension greater than $n$ in $\operatorname{Sp} A$; we will completely describe the $n$-dimensional analytic structure.

III. Analytic structure. If $A$ is a stable subalgebra of $O(K)$ we let $\Omega(A)$ denote the collection of homomorphisms $\varphi \in \operatorname{Sp} A$ for which there is a constant $c_{\varphi}>0$ such that

$$
\left|\varphi\left(D_{\sigma}^{\alpha} \mathrm{f}\right)\right| \leqslant \alpha ! c_{\varphi}^{|\alpha|}\|f\|_{K}
$$

for each $\mathrm{f} \in \boldsymbol{A}$ and each multi-index $\alpha$, where, as usual, we have written $|\alpha|$ $=\alpha_{1}+\alpha_{2}+\cdots+\alpha_{n}, \alpha !=\alpha_{1} ! \alpha_{2} ! \cdots \alpha_{n} !$ and

$$
\|\mathrm{f}\|_{K}=\sup \{|\mathrm{f}(x)|: x \in K\} \text {. }
$$

Our first result is as follows.

THEOREM 1. If $A$ is a stable subalgebra of $O(K)$ then $\Omega(A)$ admits the structure of an n-dimensional complex-analytic manifold in such a way that:

(i) the manifold topology is finer that the weak-* topology;

(ii) $\hat{\mathrm{f}} \Omega(A)$ is analytic for each $\mathbf{f} \epsilon A$; 
(iii) $\hat{\sigma} \mid \Omega(A)$ is a local analytic isomorphism, so that $(\Omega(A), \hat{\sigma} \mid \Omega(A))$ is a Riemann domain;

(iv) for each $\mathrm{f} \epsilon A$, each $\varphi \in \Omega(A)$ and each multi-index $\alpha,\left(D_{\sigma}^{\alpha} \mathrm{f}\right)^{\wedge}(\varphi)$ $=D_{\hat{\sigma}}^{\alpha} \mathrm{f}(\varphi)\left(D_{\partial}^{\alpha}\right.$ denotes differentiation with respect to $\left.\delta\right) ;$

(v) each component of $\Omega(A)$ is a Stein manifold;

(vi) $\hat{A} \mid \Omega(A)$ is dense in $\Omega(A)$ in the topology of uniform convergence on compact sets.

Proof. The argument is similar to ones employed by Bishop [4], Cartan [23] and Rossi [24], so we omit some details. Fix a homomorphism $\varphi \in \Omega(A)$. If $\mathbf{f} \epsilon A$ and $\zeta=\left(\zeta_{1}, \cdots, \zeta_{n}\right) \in \mathbf{C}^{n}$ with $|\zeta|=\max \left|\zeta_{j}\right|<c_{\varphi}^{-1}$ then we set

$$
\mathbf{f}_{\varphi}(\zeta)=\sum \varphi\left(D_{\sigma}^{\alpha} f\right) \zeta^{\alpha} / \alpha !
$$

where $\zeta^{\alpha}=\zeta_{1}^{\alpha_{1}} \zeta_{2}^{\alpha_{2}} \cdots \zeta_{n}^{\alpha_{n}}$. This series converges uniformly on compact subsets of $\left\{\zeta:|\zeta|<c_{\varphi}^{-1}\right\}$ and $\mathbf{f}_{\varphi}$ is an analytic function on that domain. If we define $\varphi_{\zeta}(f)=f_{\varphi}(\zeta)$, then straightforward calculations establish that $\varphi_{\zeta}$ is a homomorphism of $A$ into $\mathrm{C}, \varphi_{\xi}(1)=1$ and

$$
D_{z}^{\alpha} f_{\varphi}(\zeta)=\left(D_{\sigma}^{\alpha}\right)_{\varphi}(\zeta)
$$

For each $t$ with $0<t<c_{\varphi}^{-1}$, set $P_{t}=\left\{w \in \mathbf{C}^{n}:|w|<t\right\}$ and fix $\lambda \in P_{t}$. The usual Cauchy estimates for analytic functions show that there is a constant $d>0$ such that

$$
\left|D_{2}^{\beta} g(\lambda)\right| \leqslant \beta ! d^{|\beta|}\|g\|_{P_{t}}
$$

for each multi-index $\beta$ and each function $g \in O\left(P_{t}\right)$. If we substitute $f_{\varphi}$ for $g$ in (3), apply the identity in (2) and estimate the series in (1), we arrive at the following inequality

$$
\left|\varphi_{\lambda}\left(D_{\sigma}^{\beta} \mathrm{f}\right)\right| \leqslant \beta ! d^{|\beta|}\left(1-t c_{\varphi}\right)^{-n}\|\mathbf{f}\|_{K} .
$$

By considering the case $\beta=(0, \cdots, 0)$, we conclude that $\varphi_{\lambda}(\mathrm{f})=0$ if $\|\mathrm{f}\|_{K}$ $=0$ and that $\varphi_{\lambda}$ determines a continuous homomorphism on $\widetilde{A}$; hence $\left|\varphi_{\lambda}(f)\right|$ $\leqslant\|\mathrm{f}\|_{K}$. In view of Proposition 1 and the definition of $\Omega(A)$, it follows that $\varphi_{\lambda} \in \Omega(A)$. Observe that $\varphi_{0}=\varphi$ and that $\hat{\sigma}\left(\varphi_{\lambda}\right)=\lambda+\hat{\sigma}(\varphi)$.

Now for each $\psi \in \Omega(A)$, each $s$ with $0<s<c_{\psi}^{-1}$, let

$$
W(\psi, s)=\left\{\psi_{w}: w \in \mathbf{C}^{n},|w|<s\right\} .
$$

The collection $\{W(\psi, s)\}$ is a subbase for a Hausdorff topology on $\Omega(A)$ relative to which $\hat{\sigma}$ is a local homeomorphism. Thus $\hat{\sigma}$ induces on $\Omega(A)$ the structure of a complex-analytic manifold and $(\Omega(A), \partial i \Omega(A))$ is a Riemann domain. It is easy to see that the manifold topology is finer than the weak-*, and that these topologies agree on the sets $W(\psi, s)$.

For each $\mathrm{f} \in A, \varphi \in \Omega(A)$ we obtain the following power series expansion 
for $\hat{\mathbf{f}}$ on $W\left(\varphi, c_{\varphi}^{-1}\right)$ :

$$
\begin{aligned}
\hat{\mathrm{f}}\left(\varphi_{w}\right) & =\varphi_{w}(\mathrm{f})=\sum \varphi\left(D_{\sigma}^{\alpha} \mathrm{f}\right) w^{\alpha} / \alpha ! \\
& =\sum\left(D_{\sigma}^{\alpha} \mathrm{f}\right)\left[\hat{\sigma}\left(\varphi_{w}\right)-\hat{\sigma}(\varphi)\right]^{\alpha} / \alpha !
\end{aligned}
$$

so that $\hat{\mathrm{f}} \mid \Omega(A)$ is analytic. This establishes (i), (ii) and (iii); (iv) follows by straightforward calculation, which we omit.

To establish (v) and (vi), let $A^{\#}$ denote the closure of $\hat{A} \mid \Omega(A)$ in $O(\Omega(A))$. Then $A^{\#}$ is a complete, point separating algebra of analytic functions on a Riemann domain which contains the coordinates and is closed under differentiation. By a result of Bishop [4], Sp $A^{\#}$ admits the structure of a Riemann domain (with the weak-*. topology from $A^{\#}$ ) and the natural map $\Omega(A) \rightarrow \operatorname{Sp} A^{\#}$ is a homeomorphism into. Moreover, for each $\nu \in \operatorname{Sp} A^{\#}$, there is a compact set $L_{\nu} \subset \Omega(A)$ and a constant $c_{\nu}>0$ such that

$$
\left|\nu\left(D_{\widehat{\sigma}}^{\alpha} g\right)\right| \leqslant \alpha ! c_{\nu}^{|\alpha|}\|g\|_{L_{\nu}}
$$

for each $g \in A^{\#}$. But now it immediately follows that $\nu \in \Omega(A)$; i.e., Sp $A^{\#}$ $=\Omega(A)$. It then follows easily that each component of $\Omega(A)$ is holomorphically convex, and thus Stein. An application of the Oka-Weil approximation theorem yields $A^{\#}=O(\Omega(A))$, which completes the proof.

The following simple corollary gives a sufficient condition that the conclusions of Theorem 1 are not trivial.

COROLlaRY 1. If $A$ is a stable subalgebra of $O(K)$ and the interior of $K$ is not Stein (and is nonempty), then $\Omega(A) \not \subset K$.

Several remarks seem in order at this point. First, it is not always the case that $\Omega(A)$ is itself a Stein manifold; for let $C \subset[0,1]$ be a Cantor set and $A=O(K)$, where

$$
K=\left\{\left(z_{1}, z_{2}\right) \in \mathbf{C}^{2} \cdot\left|z_{1}\right|^{2}+\left|z_{2}\right|^{2}=c \in \mathbf{C}\right\} .
$$

It is easily seen that $\Omega(A)$ has uncountably many components, and is therefore not Stein (see Harvey and Wells [14] for a relevant discussion). Second, it is not always the case that the weak-* topology and the manifold topology agree, even on components of $\Omega(A)$, as the following example demonstrates.

For each positive integer $k$, let

$$
\begin{aligned}
& Y_{k}=\left\{\left(z_{1}, z_{2}\right):\left|z_{1}-1+3^{-k}\right| \leqslant 1-3^{-k},\left|z_{2}\right| \leqslant 1-3^{-k}\right\}, \\
& Y_{k}^{\prime}=\left\{\left(z_{1}, z_{2}\right):\left|z_{1}-1+3^{-k}+9^{-k}\right| \leqslant 1-3^{-k}-9^{-k},\left|z_{2}\right| \leqslant 1-3^{-k}-9^{-k}\right\},
\end{aligned}
$$

and let

$$
\begin{aligned}
& Y_{0}=\left\{\left(z_{1}, z_{2}\right):\left|z_{1}\right| \leqslant 2,\left|z_{2}\right| \leqslant 2\right\}, \\
& Y_{0}^{\prime}=\left\{\left(z_{1}, z_{2}\right):\left|z_{1}-1\right| \leqslant 1,\left|z_{2}\right| \leqslant 1\right\} .
\end{aligned}
$$


Set $L_{k}=Y_{k}-Y_{k}^{\prime}$ for $k=0,1, \cdots$. Thus each $L_{k}$ is a closed polydisk with an open polydisk removed from its side. We may connect $L_{k}$ to $L_{k+1}$ with a thin tube $T_{k}$ in such a way that these tubes are disjoint from the $Y_{k}$ 's

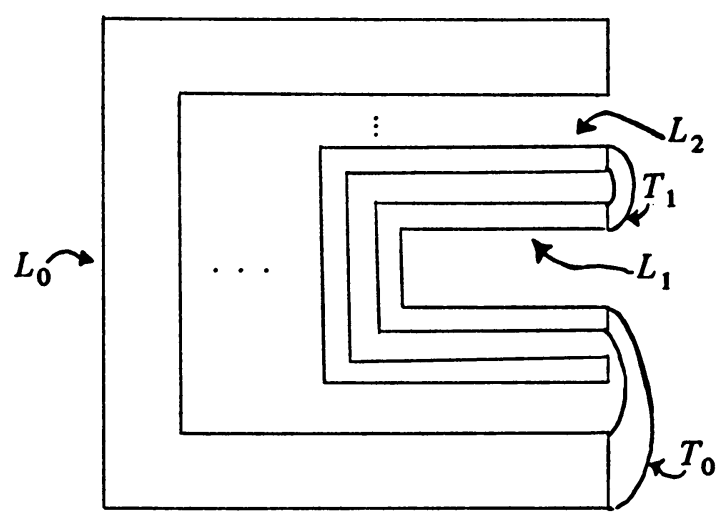

FIGURE 1

and from each other, and converge to the point $(1,1) \in L_{0}$ (see Figure 1). Set

$$
K={ }_{k=0}^{\infty} L_{k} \cup \bigcup_{k=0}^{\infty} T_{k}
$$

and let $A=O(K)$. By an argument similar to one in Gunning and Rossi [12, p. 43], it may be seen that $\Omega(A)$ is connected but infinitely sheeted over the point $(7 / 6,0)$. Moreover, we can choose a sequence $\varphi_{0}, \varphi_{1}, \cdots$ in $\Omega(A)$ such that $\hat{\sigma}\left(\varphi_{j}\right)=(7 / 6,0)$ for each $j$, but $\varphi_{j} \rightarrow \varphi_{0}$ (weak-*); i.e., the weak-* and the manifold topology do not agree. This example also displays other pathologies: $\operatorname{Sp} A$ is connected but admits no natural embedding in a Stein manifold; $\Omega(A)$ is not a weak-* open subset of $\operatorname{Sp} A$; and the Shilov boundary of $A$ contains points of $\Omega(A)$.

It is perhaps more reasonable to ask whether the manifold topology agrees (at least on connected components) with the norm topology on $\Omega(A)$ as a subset of the Banach space dual of $\widetilde{A}$. We shall have a little more to say about this following the proof of Theorem 3.

The following result assures us that we have indeed identified all the $n$-dimensional analytic structures in $\operatorname{Sp} A$.

THEOREM 2. Let $A$ be a stable subalgebra of $O(K)$ and let $X$ be a subvariety of an open set in $\mathbf{C}^{k}$, of pure dimension $n$. If $\tau: X \rightarrow \operatorname{Sp} A$ is one-one and continuous, and $\hat{\mathrm{f}} \circ \tau$ is analytic for each $\mathrm{f} \epsilon A,{ }^{\circ}$ then $X$ has no singularities and $\tau$ is an analytic isomorphism of $X$ onto an open subset of $\Omega(A)$. 
Proof. For each open set $U$ in $S$, containing $K$, let $A_{U}$ be the closure in $O(U)$ of $\{f \in O(U): f \in A\} ; A_{U}$ is then a closed, point-separating subalgebra containing the coordinates and closed under differentiation. By the theorem of Bishop [4] used earlier, $\mathrm{Sp} A_{U}$ is a Stein manifold with global local coordinate $\hat{\sigma}=\left(\hat{\sigma}_{1}, \cdots, \hat{\sigma}_{n}\right)$. If $r_{U V}^{*}: \operatorname{Sp} A_{V} \rightarrow \operatorname{Sp} A_{U}$ is the adjoint of the inclusion, then $\operatorname{Sp} A$ is the projective limit of the system $\left\{\mathrm{Sp} A_{U}, r_{U V}^{*}\right\}$. Then we have the commutative diagram of Figure 2, where $r_{V}^{*}$ is the adjoint of the inclusion and is also the projective limit map. For more details, we refer to [21].

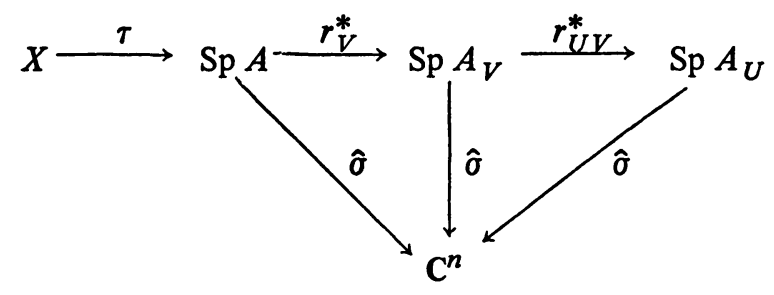

Figure 2

Fix a point $x_{0} \in X$; there is no loss in assuming that $\hat{\sigma}^{\circ} \tau\left(x_{0}\right)=0 \in \mathbf{C}^{n}$. If $E$ is a nondegenerate, connected subset of $(\hat{\sigma} \circ \tau)^{-1}(0)$, then $\tau(E)$ is a nondegenerate connected subset of $\hat{\sigma}^{-1}(0) \subset \operatorname{Sp} A$, which contradicts Proposition 2. Hence $(\partial \circ \tau)^{-1}(0)$ is totally disconnected; since it is an analytic set, it must be discrete. Let $Q_{1}$ be a relatively compact open neighborhood of $x_{0}$ such that $\bar{Q}_{1} \cap(\hat{\sigma} \circ \tau)^{-1}(0)=\left\{x_{0}\right\}$, and set $\epsilon=\min \left\{|\hat{\sigma} \circ \tau(y)|: y \epsilon \bar{Q}_{1}-Q_{1}\right\}>0$. If we set $Q=\left\{y \in Q_{1}:|\hat{\sigma} \circ \tau(y)|<\epsilon\right\}$, then it is easily seen that $\hat{\sigma} \circ \tau \mid Q$ is a proper mapping of $Q$ into $P=\left\{z \in \mathbf{C}^{n}:|z|<\epsilon\right\}$. By [12, Theorem 21, p. 108], $(Q,(\hat{\sigma} \circ \tau) \mid Q, P)$ is an analytic cover; let its sheeting number be $\lambda$.

Choose a point $z_{0} \in P$ for which $F=(\hat{\partial} \circ \tau)^{-1}\left(z_{0}\right) \cap Q$ has precisely $\lambda$ elements. There is an open set $U$ containing $K$ such that $r_{U}^{*} \circ \tau(F)$ also has $\lambda$ elements. Observe that $r_{U}^{*} \circ \tau$ is continuous and $\hat{\sigma}^{\circ} r_{U}^{*} \circ \tau=\hat{\sigma} \circ \tau$ is analytic; since $\hat{\sigma}$ determines the analytic structure of $\mathrm{Sp} A_{U}$, it follows that $r_{U}^{*} \circ \tau$ is analytic. An argument similar to that of the preceding paragraph shows that $r_{U}^{*}{ }^{\circ} \tau$ is locally proper, and hence an open map. If we set $Q^{\prime \prime}=r_{U}^{*}{ }^{\circ} \tau(Q)$, it is easy to check that $\hat{\sigma} \mid Q^{\prime \prime}$ is also proper and hence that $\left(Q^{\prime \prime}, \hat{\sigma} \mid Q^{\prime \prime}, P\right)$ is an analytic cover. But $\hat{\sigma}$ is a local homeomorphism so that $\hat{\sigma} \mid Q^{\prime \prime}$ is then a covering map; since $P$ is simply connected, the sheeting number of $\hat{\sigma} \mid Q^{\prime \prime}$ must be 1 . But $F^{\prime \prime}=r_{U}^{*} \cdot \tau(F)$ is a subset of $Q^{\prime \prime}$ containing $\lambda$ elements, and $\hat{\sigma}\left(F^{\prime \prime}\right)=$ $z_{0}$ so that we necessarily have $\lambda=1$ also. Thus we may conclude: $r_{U}^{*} \circ \tau \mid Q$ is an analytic homeomorphism onto $Q^{\prime \prime}, \hat{\sigma}^{\circ} \tau \mid Q$ is an analytic homeomorphism 
onto $P$, and $\hat{\sigma} \mid Q^{\prime \prime}$ is an analytic homeomorphism onto $P$. In particular, $Q$ contains no singularities of $X$.

Let $L_{\delta}$ be a compact subset of $Q$ such that $\hat{\sigma} \circ \tau$ maps $L_{\delta}$ homeomorphically onto $P_{\delta}=\left\{z \in \mathrm{C}^{n}:|z| \leqslant \delta\right\}$, where $0<\delta<\epsilon$. Let $V$ be an arbitrary open set in $S$ which contains $K$. It is easy to check that $r_{V}^{*} \circ \tau \mid Q$ is an analytic homeomorphism, as is $\hat{\sigma} \mid Q_{V}$, where $Q_{V}=r_{V}^{*} \circ \tau(Q)$. If $L_{\delta}^{\prime \prime}=$ $r_{V}^{*} \circ \tau\left(L_{\delta}\right)$, then $L_{\delta}^{\prime \prime}$ is a compact subset of $\operatorname{Sp} A_{V}$ and $\hat{\sigma}$ maps $L_{\delta}^{\prime \prime}$ homeomorphically onto $P_{\delta}$. Observe that $y_{0}=r_{V}^{*} \cdot \tau\left(x_{0}\right)$ is in the interior of $L_{\delta}^{\prime \prime}$. The usual Cauchy estimates show that

$$
\left|D_{\hat{\sigma}}^{\alpha} g\left(y_{0}\right)\right| \leqslant \alpha !\left(\delta^{-1}\right)^{|\alpha|}\|g\|_{L_{\delta}^{\prime \prime}}
$$

for each multi-index $\alpha$ and each $g \in O\left(\operatorname{Sp} A_{V}\right)$. As in the proof of Theorem 1, we see that $D_{\hat{\sigma}}^{\alpha} \hat{h}\left(y_{0}\right)=\left(D_{\sigma}^{\alpha} h\right)^{\wedge}\left(y_{0}\right)$ for each $h \in A_{V}$. If we set $\varphi=\tau\left(x_{0}\right)$ and $L_{\delta}^{\prime}=\tau\left(L_{\delta}\right)$, then we have:

$$
\begin{aligned}
\left|\varphi\left(D_{\sigma}^{\alpha} \mathrm{f}\right)\right| & =\left|\left(D_{\sigma}^{\alpha} f\right)^{\hat{\gamma}}\left(y_{0}\right)\right|=\left|D_{\hat{\sigma}}^{\alpha} \hat{f}\left(y_{0}\right)\right| \\
& \leqslant \alpha !\left(\delta^{-1}\right)^{|\alpha|}\|\hat{f}\|_{L_{\delta}^{\prime \prime}} \leqslant \alpha !\left(\delta^{-1}\right)^{|\alpha|}\left\|\hat{f} \circ r_{V}^{*}\right\|_{L_{\delta}^{\prime}} \leqslant \alpha !\left(\delta^{-1}\right)^{|\alpha|}\|\hat{f}\|_{K}
\end{aligned}
$$

for each multi-index $\alpha$ and each $f \in O(V)$ for which $\mathrm{f} \epsilon A$. Since $V$ is arbitrary, it follows that $\varphi=\tau\left(x_{0}\right) \in \Omega(A)$; hence $\tau(X) \subset \Omega(A)$.

It only remains to show that $\tau$ is continuous when $\Omega(A)$ has its manifold topology, for then the analyticity of $\hat{\sigma} \circ \tau$ will yield that $\tau$ is analytic, one-one, and hence a homeomorphism. Continuity of $\tau$ will follow if we can show that $\tau(Q)=W(\varphi, \epsilon)$, since as was observed in the proof of Theorem 1, the manifold and weak-* topologies agree on sets of the form $W(\varphi, \epsilon)$. Observe first of all that the inequality (6) is valid for all $\delta<\epsilon$ and hence is valid for $\delta=$ $\epsilon$; i.e.

$$
\left|\varphi\left(D_{\sigma}^{\alpha} \mathrm{f}\right)\right| \leqslant \alpha !\left(\epsilon^{-1}\right)^{|\alpha|}\|\hat{\mathbf{f}}\|_{K}
$$

so that $c_{\varphi} \leqslant \epsilon^{-1}$ and $c_{\varphi}^{-1} \geqslant \epsilon$. Let $q \epsilon Q$ and set $\zeta=\hat{\sigma} \cdot \tau(q)$; we claim $\tau(q)=\varphi_{\zeta}$. For, if $f \in O(V)$ and $\mathbf{f} \epsilon A$, then we can expand $\hat{f}$ in a power series near $y_{0}=r_{V}^{*} \circ \tau\left(x_{0}\right)$ :

$$
\hat{f}(t)=\sum\left(D_{\hat{\sigma}}^{\alpha} \hat{f}\left(y_{0}\right)\right)\left(\hat{\sigma}(t)-\hat{\sigma}\left(y_{0}\right)\right)^{\alpha} / \alpha !
$$

Since $\hat{\sigma}$ maps $Q_{V}$ homeomorphically onto the polydisk $P \subset \mathrm{C}^{n}$, this power series converges on $Q_{V}$, and in particular at $y_{1}=r_{V}^{*} \circ \tau(q)$. A direct calculation now shows that $\hat{f}\left(y_{1}\right)=\hat{f}(\tau(q))=\varphi_{\xi}(f)$. Since $V$ and $\zeta$ are arbitrary, we have $\tau(Q)=W(\varphi, \epsilon)$, which completes the proof.

A slight modification of the latter part of the above argument leads to the following topological characterization of $\Omega(A)$. 
COROLlaRY 2. If $\varphi \in \operatorname{Sp} A$, then $\varphi \in \Omega(A)$ if and only if there are a set $W \subset \operatorname{Sp} A$, containing $\varphi$, and germs $\mathrm{f}_{1}, \mathrm{f}_{2}, \cdots, \mathrm{f}_{n} \in A$ such that $\left(\hat{\mathbf{f}}_{1}, \hat{\mathbf{f}}_{2}, \cdots, \hat{\mathbf{f}}_{n}\right)$ is a homeomorphism of $W$ (in the weak-* topology) onto an open subset of $\mathbf{C}^{n}$.

By mimicking the above arguments, we can derive (less definitive) results for analytic structure of lower dimension. For $1 \leqslant k \leqslant n$, let $\Omega_{k}(A)$ denote the set of homeomorphisms $\varphi \in \operatorname{Sp} A$ for which there is a constant $c>0$ such that $\left|\varphi\left(D_{\sigma}^{\alpha} \mathrm{f}\right)\right| \leqslant \alpha ! c^{|\alpha|}\|\mathrm{f}\|_{K}$ for each $\mathbf{f} \epsilon A$, each multi-index $\alpha$ of the form $\alpha=\left(\alpha_{1}, \alpha_{2}, \cdots, \alpha_{k}, 0, \cdots, 0\right)$. Then we have the following analogs of the above results; the proofs are virtually identical and are omitted.

THEOREM 1'. If $A$ is a stable subalgebra of $O(K)$ then $\Omega_{k}(A)$ admits the structure of a $k$-dimensional complex-analytic manifold in such a way that:

(i) the manifold topology is finer than the weak-* topology;

(ii) $\hat{\mathrm{f}} \mid \Omega_{k}(A)$ is analytic for each $f \in A$;

(iii) $\mu=\left(\hat{\sigma}_{1}, \cdots, \hat{\sigma}_{k}\right)$ is a local analytic isomorphism, so that $\left(\Omega_{k}(A), \mu\right)$ is a Riemann domain;

(iv) for each $\mathrm{f} \epsilon A$ each $\varphi \in \Omega_{k}(A)$ and each $\alpha=\left(\alpha_{1}, \cdots, \alpha_{k}, 0, \cdots, 0\right)$, $D_{\mu}^{\alpha} \hat{\mathrm{f}}(\varphi)=\left(D_{\sigma}^{\alpha} \mathrm{f}\right)^{\wedge}(\varphi)$

(v) each component of $\Omega_{k}(A)$ is a Stein manifold;

(vi) $\hat{A} \mid \Omega_{k}(A)$ is dense in $\Omega_{k}(A)$ in the topology of uniform convergence on compact sets;

(vii) for $k<j \leqslant n, \hat{\sigma}_{j}$ is constant on each component of $\Omega_{k}(A)$.

THEOREM 2'. Let $A$ be a stable subalgebra of $O(K)$ and let $X$ be a subvariety of an open set in $\mathrm{C}^{j}$ of pure dimension $k$. If $\tau: X \rightarrow \operatorname{Sp} A$ is one-one and continuous, $\hat{\mathrm{f}} \circ \tau$ is analytic for each $\mathrm{f} \in A$ and $\hat{\sigma}_{j} \cdot \tau$ is constant for $k<j \leqslant n$, then $X$ has no singularities and $\tau$ is an analytic isomorphism of $X$ onto an open subset of $\Omega_{k}(A)$.

COROLlaRY $2^{\prime}$. If $\varphi \in \operatorname{Sp} A$ then $\varphi \in \Omega_{k}(A)$ if and only if there are a set $W \subset \operatorname{Sp} A$, containing $\varphi$ and germs $\mathbf{f}_{1}, \cdots, \hat{f}_{k} \in A$ such that $\left(\hat{f}_{1}, \cdots, \hat{\mathbf{f}}_{k}\right)$ is a homeomorphism of $W$ (in the weak-* topology) onto an open subset of $\mathrm{C}^{k}$, and $\hat{\sigma}_{j} \mid W$ is constant for $k<j \leqslant n$.

IV. Point derivations. If $B$ is a commutative topological algebra with identity, and $\varphi \in \operatorname{Sp} B$, we recall that a point derivation on $B$ at $\varphi$ is a linear functional $T: B \rightarrow C$ such that

$$
T\left(b_{1} b_{2}\right)=\varphi\left(b_{1}\right) T\left(b_{2}\right)+T\left(b_{1}\right) \varphi\left(b_{2}\right)
$$

for each $b_{1}, b_{2} \in B$. Equivalently, we may identify a point derivation with an element of the algebraic dual space of $(\operatorname{Ker} \varphi) /(\operatorname{Ker} \varphi)^{2}$. As Browder [7], [8] has shown, there is an intimate and remarkable connection between point derivations on Banach algebras and analytic structure in the spectrum. In the present 
context, we have the following result concerning continuous point derivations on stable function algebras.

THEOREM 3. Let $A$ be a stable subalgebra of $O(K), \varphi \in \operatorname{Sp} A=\operatorname{Sp} \tilde{A}$, and let $D_{\varphi}$ denote the complex vector space of continuous point derivations on $\tilde{A}$ at $\varphi$. Then the dimension of $D_{\varphi}$ (over C) does not exceed $n^{2}$, if $\varphi \in \Omega(A)$, the dimension is precisely $n$.

The proof will utilize the following lemma, which may be proved via a standard application of Cartan's Theorem B (a similar lemma appears in [22]).

Lemma. Let $M$ be a Stein manifold of dimension $n$, let $m$ be a point of $M$, and let $\mu_{1}, \mu_{2}, \cdots, \mu_{n} \in O(M)$, give coordinates at $m$ For each $f \in O(M)$, there are complex numbers $\lambda_{1}, \lambda_{2}, \cdots, \lambda_{n}$, and functions $g_{1}, \cdots, g_{2 n+1}, h_{1}, \cdots, h_{2 n+1}$ in $O(M)$, each vanishing at $m$, such that

$$
f=f(m)+\sum_{i=1}^{n} \lambda_{1}\left(\mu_{i}-\mu_{i}(m)\right)+\sum_{j=1}^{2 n+1} g_{j} h_{j}
$$

Proof of Theorem 3. There is evidently no loss of generality in assuming that $A$ is closed in $O(K)$. Then $r_{U}^{-1}(A)$ is closed in $O(U)$ for each open set $U$ containing $K$; i.e., $A_{U}=\{f \in O(U)$ : f $\epsilon A\}$. If $T$ is a continuous point derivation on $\widetilde{A}$ at $\varphi$, then $T$ clearly induces a point derivation $T_{U}$ on $A_{U}$ at $\varphi_{U}=r_{U}^{*}(\varphi)$. Recall from Bishop's theorem [4] that $\mathrm{Sp} A_{U}$ is a Stein manifold with global coordinate $\left(\hat{\sigma}_{1}, \cdots, \hat{\partial}_{n}\right)$, and that $\hat{A}_{U}=O\left(\operatorname{Sp} A_{U}\right)$. Thus if $J=$ kernel $\varphi_{U}$, the lemma implies that $J / J^{2}$ is $n$-dimensional, as its dual. Hence there are $\lambda_{1}, \cdots, \lambda_{n} \in \mathbf{C}$ such that $T_{U}=\Sigma_{i=1}^{n} \lambda_{i}\left(\varphi_{U} \circ \partial / \partial \sigma_{j}\right)$ on $A_{U}$; since $T_{U}=T_{V} \circ r_{U V}$, it follows that the $\lambda_{i}$ are independent of the choice of $U$. Since $T$ is continuous and the natural map $A \rightarrow \widetilde{A}$ has dense range, it follows that the map $T \rightarrow\left(\lambda_{1}, \cdots, \lambda_{n}\right): D_{\varphi} \rightarrow C^{n}$ is well defined, one-one and linear. Hence the dimension of $D_{\varphi}$ does not exceed $n$, as asserted.

If $\varphi \in \Omega(A)$, then the maps $g \mapsto \partial \hat{g}(\varphi) / \partial \hat{\sigma}_{j}(j=1,2, \cdots, n)$ are defined for each $g \in \tilde{A}$, (since functions in $\widetilde{A}^{\wedge}$ are uniform limits of functions in $\hat{A}$, they are certainly analytic on $\Omega(A)$ ) and are easily seen to be linearly independent, continuous point derivations on $\tilde{A}$ at $\varphi$.

Unfortunately, the dimension of $D_{\varphi}$ does not characterize those homomorphisms $\varphi$ which belong to $\Omega(A)$, even in the case $n=1$. Hallstrom [13] has constructed examples in which $\operatorname{dim}_{\mathrm{C}} D_{\varphi}=n=1$ for almost all points $\varphi \in \operatorname{Sp} A$ while $\Omega(A)=\varnothing$.

If it could be shown that, for each $\varphi \in \Omega(A)$, the space of (not necessarily continuous) point derivations on $\widetilde{A}$ at $\varphi$ were finite dimensional, then it would 
follow by results of Browder [8] that $\Omega(A)$ would be an open subset of $\mathrm{Sp} A$ (relative to the norm topology) and that the norm topology and manifold topology would agree on $\Omega(A)$.

\section{REFERENCES}

1. H. Alexander, Polynomial approximation and analytic structure, Duke Math. J. 38 (1971), 123-135. MR 44 \#477.

2. - Polynomial approximation and hulls of sets of finite linear measure in $\mathrm{C}^{N}$, Amer. J. Math. 93 (1971), 65-74. MR 44 \#1841.

3. E. Bishop, Analyticity in certain Banach algebras, Trans. Amer. Math. Soc. 102 (1962), 507-544. MR 25 \#5410.

4. Holomorphic completions, analytic continuation, and the interpolation of semi-norms, Ann. of Math. (2) 78 (1963), 468-500. MR 27 \#4958.

5. J.-E. Björk, Analytic structures, Papers from the Summer Gathering on Function Algebras (Aarhus, 1969), Matematisk Inst., Aarhus Univ., Aarhus, 1969, pp. 19-28. MR 41 \#827a.

6. - On analytic structures in the maximal ideal space of a function algebra, Papers from the Summer Gathering on Functional Algebras (Aarhus, 1969), Matematisk Inst., Aarhus Univ., Aarhus, 1969, pp. 29-35. MR 41 \#827b.

7. A. Browder, Point derivations on function algebras, J. Functional Analysis 1 (1967), 22-27. MR 35 \#2144.

8. Point derivations and analytic structure in the spectrum of a Banach algebra, J. Functional Analysis 7 (1971), 156-164. MR 42 \#8286.

9. D. Clayton, A local characterization of analytic structure in a commutative Banach algebra, Sympos. on Several Complex Variables (Park City, Utah, 1970), Lecture Notes in Math., vol. 184, Springer-Verlag, Berlin, 1971, pp. 10-36. MR 45 \#9138.

10. T. W. Gamelin, Uniform algebras, Prentice-Hall, Englewood Cliffs, N. J., 1969.

11. A. M. Gleason, Finitely generated ideals in Banach algebras, J. Math. Mech. 13 (1964), 125-132. MR 28 \#2458.

12. R. C. Gunning and H. Rossi, Analytic functions of several complex variables, Prentice-Hall Ser. in Modern Analysis, Prentice-Hall, Englewood Cliffs, N. J., 1965. MR 31 \#4927.

13. A. P. Hallstrom, On bounded point derivations and analytic capacity, J. Functional Analysis 4 (1969), 153-165. MR 39 \#4680.

14. F. R. Harvey and R. O. Wells, Jr., Compact holomorphically convex subsets of a Stein manifold, Trans. Amer. Math. Soc. 136 (1969), 509-516.

15. H. Rossi, The local maximum modulus principle, Ann. of Math. (2) 72 (1960). 1-11. MR 22 \#8317.

16. G. Stolzenberg, A hull with no analytic structure, J. Math. Mech. 12 (1963), 103111. MR 26 \#627.

17. - Uniform approximation on smooth curves, Acta Math. 115 (1966),

185-198. MR 33 \#307.

18. E. L. Stout, The theory of uniform algebras, Bogden and Quigley, Tarrytown-onHudson, N. Y., 1971.

19. J. Wermer, Analytic disks in maximal ideal spaces, Amer. J. Math. 86 (1964), 161-170. MR 28 \#5355. 
20, W. Zame, Algebras of analytic functions in the plane, Pacific J. Math. 42 (1972), 811-819.

21. - Algebras of analytic germs, Trans. Amer. Math. Soc. 174 (1972), 275288. MR 47 \#2099.

22. - Homomorphisms of rings of germs of analytic functions, Proc. Amer. Math. Soc. 33 (1972), 411-414.

23. Séminaire H. Cartan, 1951/52, Ecole Normale Supérieure, Paris, 1952. MR 16, 233.

24. H. Rossi, On envelopes of holomorphy, Comm. Pure Appl. Math. 16 (1963), 917. MR $26 \# 6436$.

DEPARTMENT OF MATHEMATICS, STATE UNIVERSITY OF NEW YORK AT BUFFALO, AMHERST, NEW YORK 14226 